\title{
MEDIDAS DE SEGUIMIENTO Y CONTROL PARA LA MAQUINARIA UTILIZADA EN LOS PROCESOS DE EXTRACCIÓN MINERA
}

\section{MONITORING AND CONTROL MEASURES FOR THE MACHINERY USED IN THE MINING EXTRACTION PROCESSES}

\author{
CD. David Nicolas Achicanoy Caicedo² \\ CD. Alexis Arley Acosta Afanador ${ }^{3}$ \\ CD. Gilmar Fabian Albornoz Perea 4 \\ Dra. Nubia Edith Céspedes Prieto ${ }^{5}$ \\ Dr. Ernesto Fajardo Pascagaza. ${ }^{6}$
}

\section{RESUMEN}

Esta investigación se basa en información suministrada por el Ministerio de Defensa
Nacional, la cual describe que la explotación ilícita de yacimientos mineros está presente en veinticinco departamentos del territorio nacional

$1 \quad$ Artículo producto de la investigación realizada al interior del Grupo de Investigación ECSAN en desarrollo de la Especialización en Servicio de Policía, en torno a la relación al impacto ambiental ocasionado por la destrucción de la maquinaria, de acuerdo a la resolución 06706 del 2007 en la línea construcción de paz y medio ambiente.

$2 \quad$ Cadete de la escuela de cadetes de Policía "General Francisco de Paula Santander", Abogado, E-mal: david.achicanoy@correo.policia.gov.co._Orcid: https://orcid.org/0000-0003-2669-7283

3 Cadete de la escuela de cadetes de Policía "General Francisco de Paula Santander", Ingeniero Ambiental, E-mal: alexis.acostaa@correo.policia.gov.co Orcid: https://orcid.org/0000-0001-5905-1935

4 Cadete de la escuela de cadetes de Policía "General Francisco de Paula Santander", Ingeniero Ambiental, E-mal: gilmar.albornoz@correo.policia.gov.co Orcid: https://orcid.org/0000-0002-8326-5869

5 Doctora en Ciencias, Magister en Ciencias Químicas, Licenciada en Química y Biología, Ingeniera Ambiental. Docente de la ECSAN. Integrante del Grupo de Investigación ECSAN Categorizado por Colciencias en C. Investigadora Categorizada por Colciencias como Investigadora Asociada. Correo electrónico: necespedesp@unal.edu.co Orcid: https://orcid.org/0000-00016334-989X

6 Doctorando en Educación, Doctorando en Filosofía, Magíster en Filosofía y Magíster en Educación. Docente del Departamento de Humanidades y Formación Integral de la USTA. Integrante del Grupo de Investigación ALETHEIA Categorizado Colciencias en A1. Investigador Categorizado por Colciencias como Investigador Asociado. Correo electrónico: ernesto- fajardo@ usantotomas.edu.co Orcid: https://orcid.org/0000-0003-1168-9512 
los cuales son los que sufren la mayor afectación ambiental y social. De acuerdo a lo anterior es necesario elaborar un plan de seguimiento y control para la maquinaria usada en la minería en Colombia y contribuir con parámetros para el uso adecuado de esta. Resulta imperioso el análisis de legislación vigente que brinde las herramientas para cumplir este propósito. Si bien el direccionamiento de la investigación lleva a trabajar en conjunto con la Policía Nacional, esto no deja de lado la colaboración con otras instituciones públicas, como los son las Corporaciones Autónomas Regionales y la Dirección de Impuestos y Aduanas Nacionales

PALABRAS CLAVES: minería; medio ambiente; maquinaria; legislación; policía; público

\section{SUMMARY}

This investigation is based on information provided by the Ministry of National Defense, which describes that the illicit exploitation of mining deposits is present in twenty-five departments of the national territory, which are the ones that suffer the greatest environmental and social damage. According to the above, it is necessary to prepare a monitoring and control plan for the machinery used in mining in Colombia and contribute parameters for the proper use of it. The normative analysis that provides the tools to fulfill this purpose is imperative. Although the direction of the investigation leads to working together with the National Police, this does not neglect collaboration with other public institutions, such as the Regional Autonomous Corporations and Directorate of National Taxes and Customs.

KEY WORDS: mining; environment; machinery; legislation; police; public

\section{INTRODUCCIÓN}

Uno de los principios que guían la labor de la Policía Nacional ha sido siempre la protección, cuidado y preservación del medio ambiente, y aunque el campo de acción de la institución es la atención de este tipo de asuntos, pareciera en principio que se ve limitada solamente a suspender o dar por terminada la conducta ilegal, visto lo anterior observamos como esta problemática trasciende en el tiempo debido a que no se tiene claro quienes, como, en qué momento y de qué manera se debe intervenir la maquinaria abandonada en distintos parajes del territorio, sin una debida disposición final. Para ello es fundamental la cooperación entre instituciones que cuentan con el mismo objetivo de protección y cuidado ambiental, esta labor no puede ser solo responsabilidad de la Policía.

Identificar un plan de seguimiento, control y disposición final para la maquinaria utilizada en los procesos de extracción minera, se trata del objetivo final de la presente investigación. De alcanzarse las metas del proyecto planteado supondría una mejora considerable en la actividad de minería en nuestro país. Muy difícilmente se dará por resuelta esta problemática, pero se trata de un primer paso para lograrlo. Aunando a lo anterior se cuenta con la posibilidad de dar a la maquinaria incautada un propósito distinto para el cual venía siendo utilizada, bajo el entendido de que no debe ser destruida al momento de realizar las intervenciones policiales, lo cual genera múltiples afectaciones al medio ambiente, en su lugar, destinarla a las zonas del país en las cuales pueda ser aprovechada en beneficio de distintas obras en favor de la ciudadanía.

\section{DESARROLLO}

Colombia es un país donde las prácticas ilegales trascienden varios aspectos de la sociedad y su modo de vida. Fenómenos como el narcotráfico o el conflicto armado han sido los grandes precursores de estas malas prácticas. Bien es sabido que muchas de estas conductas afectan directa e indirectamente el medio ambiente y los recursos naturales del país y gran parte de esta catástrofe la han producido aquellos 
actores dedicados a la minería ilegal, fenómeno que se presenta debido a diferentes tipos de consideraciones, que no es objetivo determinar el porqué de ellos en el presente artículo, ni tampoco dar soluciones o tratar de visualizar como acabar con este tipo de conductas ilegales, el objetivo es determinar en primer término cuales son los protocolos o procedimientos adecuados para la adquisición de maquinaria y el buen empleo de esta en la extracción del recurso minero del país, si se hace realmente un control y vigilancia adecuado de los permisos mineros y fundamentalmente se pretende vislumbrar un camino o una hoja de ruta a la problemática planteada por la Dirección de Carabineros de la Policía Nacional de Colombia sobre la destinación final de la maquinaria ilegal e insumos utilizados en estos procesos de extracción minera ilegales, y que luego de suspendida la actividad, permanecen en las zonas intervenidas contribuyendo al deterioro ambiental con su sola presencia en el área.

Remitirnos a las apreciaciones y discusiones que sobre el tema se han dado es crucial para vislumbrar un panorama acerca de cuáles son las opciones con las que se cuenta realmente para afrontar la problemática planteada. En primer lugar y tratando de contextualizar la realidad de la minería ilegal y su impacto ambiental en el país se ha dicho por parte de las instituciones la cuales precisan de manera muy clara que la problemática en general se manifiesta obviamente en los distintos escenarios de explotación minera pero que adquieren mayor trascendencia cuando la ilegalidad, informalidad y falta de control gubernamental contribuyen al deterioro de las relaciones entre los distintos actores involucrados en estas prácticas, los cuales solamente ven abusos y falta de oportunidades, lo que desemboca en un mayor perjuicio al medio ambiente. (Informe Preventivo Minería llegal, Procuraduría General de la Nación, 2016)
Tratando de llevar el análisis hacia las regiones se encuentra con que los departamentos del Choco y Valle del Cauca son unos de los más afectados, al respecto afirma el artículo "Los Impactos Socioambientales en el Pacífico", que la explotación ilegal derivada del uso de maquinaria especializada en Colombia se encuentra en el orden del $63 \%$, siendo de mayor atractivo la minería del oro y distintos materiales los cuales para su extracción requieren que se cause un constante y preocupante balance negativo sobre el medio ambiente. Se hace ya mención al uso de maquinaria, al respecto y en una aproximación al origen de los problemas ocasionados por la minería ilegal asegurando que estas son producto de "La utilización de maquinaria (retroexcavadoras y dragas de succión); carencia de estudios de exploración; desconocimiento de técnicas de explotación y beneficio" (Informe especial de la Contraloria (Mineria llegal), 2013).

Para concluir y manera de dar precisión a las cifras, se consagra que "El Sistema de Monitoreo Antinarcóticos de la Policía (SIMA), que utiliza alta tecnología para ubicar las zonas con cultivos ilícitos y minería clandestina, ha identificado en todo el país 6.330 puntos donde se saca oro de aluvión. Sus reportes de inteligencia señalan que hay 95.000 hectáreas "con total afectación" por efectos de la extracción sin control. Chocó (40.780 hectáreas), Antioquia (35.581 hectáreas), Bolívar (8.629) y Córdoba (5.291) tienen los mayores niveles de daño." (La mineria sin control de la defensoria del pueblo, 2015)

De acuerdo con lo anterior se puede observar que la problemática ya ha sido identificada y se cuenta con datos y cifras claras al respecto, y la legislación nacional también ha abordado es asunto a través del Decreto Numero 2235 de 2012 - Relación con el uso de maquinaria pesada y sus partes en actividades mineras, el cual aclara cuál es la relación con el uso de maquinaria pesada y sus partes en actividades 
mineras sin las autorizaciones y exigencias previstas en la ley. Este decreto abarca todo el territorio nacional, especialmente las zonas que se ven afectadas por el uso de maquinaria pesada y sus partes o derivados en actividades mineras las cuales carecen de las respectivas autorizaciones y exigencias previstas en la normatividad. (Decreto 2235, 2012)

Se tiene hasta este punto certeza acerca de que una de las principales razones por las cuales la minería ilegal persiste en el país es el uso de la maquinaria, observación que resulta obvia ya que si no se contara con los medios para extraer los minerales del suelo y subsuelo, simplemente no se encontraría el país ante un escenario cada vez más complejo con respecto a las prácticas ilegales en la minería, pero que es el punto focal de la presente investigación y es un tema ya tratado incluso por la comunidad internacional, afirmando que cuando se despliegan actividades de minería ya sea por parte de personas naturales o jurídicas, pero las cuales no cuentan con las respectivas autorizaciones de orden legal, tales como títulos mineros o licencias ambientales, esta última por lo general requerida a la hora de explotar recursos naturales, procede la medida de destrucción de la maquinaria y sus partes tal como se encuentra enmarcado en la Decisión 774 de 2012 de la Comunidad Andina de Naciones, más allá de quien sea el titular ya sea de la maquinaria, como de los permisos y autorizaciones. (Agencia Nacional de Mineria, 2014).

La Policía Nacional es la autoridad competente para ejecutar la medida de destrucción de la maquinaria pesada y sus partes, que esté siendo utilizada en actividades de exploración o explotación de minerales de manera ilegal. (Sistema unico de informacion normativo, 2012).

No obstante lo planteado acerca de la labor de la Policía Nacional se tiene definido claramente que el deber de cuidado y protección del medio ambiente, no le corresponde a la institución policial, ni a otras instituciones de manera individual, la labor debe ser conjunta y articulada, no puede pasarse por alto entonces el aporte de entidades como las CAR Corporaciones Autónomas Regionales, para la muestra un estudio realizado por Corpoamazonia denominado; “Análisis sobre la viabilidad de reglamentar el uso, movilización y transporte de la principal maquinaria pesada y equipos empleados en la minería ilegal de oro en la jurisdicción de Corpoamazonia", el cual buscó diseñar una medida administrativa de manejo ambiental para la prevención y el control de las actividades de minería ilegal de oro a partir de la maquinaria y equipos utilizados en la exploración y explotación de este mineral, en los departamentos de Amazonas, Caquetá y Putumayo. (Instituto Amazónico de investigación Cientificas, 2018)

Así como el tratamiento de esta problemática se ha visto trabajado así sea de manera precaria al interior del país, este tipo de asuntos tienen sus propias aristas y puntos de impacto a nivel internacional, puede observarse como referencia una investigación desarrollada en el vecino país del Ecuador titulada; “Necesidad de reformar el art.- 57 de la ley orgánica reformatoria a la ley de minería, sobre la afectación social que produce la destrucción, demolición, inutilización o neutralización de maquinaria equipos, insumos y vehículos utilizados en actividades ilegales de explotación minera", el cual trata la problemática social que surge a partir de la aplicación de las leyes nacionales (Ecuador), que han logrado la destrucción de gran cantidad de maquinaria decomisada en actividades de explotación minera ilegal; destacando así que como consecuencia principal se ha venido afectando indirecta y significativamente a todo el país, puesto que se les niega a los ciudadanos el derecho de aprovechar estos recursos materiales en obras de múltiples beneficios para los mismos; es por ello que se hace necesario reformar la legislación minera en cuanto al 
tema señalado de acuerdo a la realidad social que engloba esta problemática.

Esta investigación realizada en Ecuador arroja claramente una de las opciones a tenerse en cuenta al momento de incautar maquinaria utilizada en la minería ilegal, consistente en otorgarle la propiedad de las mismas a la ciudadanía. Para el caso colombiano se manejaría de manera distinta, alternativa la cual se abordará más adelante. (Torres Jimenez, 2015)

La información recolectada en conjunto de estas investigaciones y normatividad permite un acercamiento a los distintos factores que originan la problemática planteada. Adoptar los primeros pasos para la consecución del objetivo propuesto el cual es identificar un plan de seguimiento, control y disposición final para la maquinaria utilizada en los procesos de extracción minera, especialmente la incautada por la Policía Nacional de Colombia en el contexto de su misión institucional de cuidado y protección del medio ambiente articulando esfuerzos con aquellas instituciones enfocadas al mismo objetivo. Analizando la realidad colombiana se encuentra un panorama llenado de tecnicismos y obstáculos burocráticos engorrosos para la obtención de permisos para ejercer la minería. Pero el problema planteado radica en las comunidades más vulnerables que encuentran en la minería ilegal su sustento de vida, es a ellos entonces donde deben ir enfocados los protocolos planteados de seguimiento, control y disposición final.

\section{MÉTODOS}

La presente investigación es de carácter Cualitativo, no se parte de premisas no desarrolladas, sino que por el contrario se cuenta con una serie de datos aportados por la Dirección de Carabineros y UNIMIL (Unidad Nacional contra la Minería Ilegal y Antiterrorismol en cuanto a la problemática sobre el uso y destinación final de la maquinaria utilizada para explotar ilegalmente los recursos mineros del país. Se plantean opciones de cómo abordar el problema desde el punto de vista jurídico y ambiental.

Aplicar un enfoque cualitativo al estudio de distintos fenómenos, parte de la premisa de no trastocar o distorsionar el objeto y a tener una mirada amplia del problema. Al emplear este método debe tenerse sumamente en cuenta la importancia de un análisis en un contexto generalizado del objeto social investigado, entendido como un complejo sistema donde cada elemento que lo compone se encentra interrelacionado uno con otro. Por lo tanto, apartar la mirada a esta realidad y fragmentar los componentes que la integran solo dejaría como consecuencia una visión parcial, alejada de la realidad e incompleta. (Investigación Cualitativa, 2007)

La investigación cualitativa cuenta con diferentes métodos, técnicas y recursos para indagar distintos aspectos Es importante el aporte de este tipo de investigación a los estudios en casos de extracción minera debido a que identifican que produce esto a nivel social y ambiental por otra parte, aporta a esta investigación información a la dirección de carabineros que es de vital importancia para minimizar estas actividades ilegales en todo el territorio nacional.

\section{RESULTADOS}

Realizar el análisis de como la maquinaria utilizada en minería ilegal se ha tornado un problema para la Policía Nacional, debido a la dificultad que representa su disposición final, es importante mencionar que de acuerdo a lo estipulado en el Decreto No. 2235 del 30 de octubre de 2017, emanado por el Ministerio de Defensa Nacional, el cual se refiere al control de la minería ilegal en el territorio colombiano, se puntualizan las pautas para la destrucción de maquinaria pesada y sus componentes que 
tengan como fin la utilización de la misma en actividades de exploración, construcción, montaje y explotación de minerales y lo requerido por la ley.

En concordancia y debido al fenómeno de la minería ilegal y su deterioro a elementos ambientales económicos y sociales los planes de desarrollo nacionales estructuran como prioridad prohibición en todo el territorio nacional el empleo de maquinaria pesada en las actividades mineras sin título correspondiente y los requisitos importantes como lo son el programa de trabajos y obras y su respectiva viabilidad ambiental (Plan de Manejo Ambiental o Licencia Ambiental) según el tipo de proyecto minero a tratar.

Es de aclarar que al poner en marcha alguna de las etapas que estructuran o contiene el titulo minero (Exploración, construcción y montaje, explotación) sin ninguna viabilidad ambiental trae consigo consecuencias como el deterioro de los componentes bióticos y abióticos del medio ambiente, como lo es la pérdida del paisaje, perdida del recurso hídrico fauna y flora, y poniendo en riesgo la seguridad del territorio y su soberanía, teniendo en cuenta que la actividad ilegal no generaliza la importancia de las medidas de mitigación y corrección que garanticen lo reversible a los impactos ambientales derivados de la misma, obviando también la problemática del uso inadecuado de sustancias altamente peligrosas y toxicas, no dejando atrás factores de riesgos ambientales y sociales como lo es el uso descontrolado e inapropiado de maquinaria pesada como lo son la buldócer, retroexcavadoras, y dragas, seguido al transporte y licencias para la importación de la misma.

Es de suma importancia tener en cuenta la importancia del objeto del Decreto 2261 del 2 de noviembre de 2012, el cual estipula la obligatoriedad de obtener la licencia para la importación de maquinaria especializada y el transporte de la misma en el territorio nacional para diferentes actividades entre ellas la minería, la cual establece en su artículo primero los siguientes requisitos:

1. Que el importador de la maquinaria obtenga la autorización de la Dirección de Impuestos y Aduanas Nacionales, acreditando previamente lo siguiente los siguientes presupuestos:

$\checkmark$ Estar inscrito en el Registro Único Tributario, con condición de declarante del impuesto de renta, responsable del régimen común del impuesto sobre las ventas, y usuario aduanero importador;

$\checkmark$ Diligenciar el formulario oficial a través del servicio informático de la DIAN, la solicitud de importador con los datos de su domicilio, actividad económica, sub partidas arancelarias de los bienes a importar, destino y uso;

$\checkmark$ Estar domiciliado o representado legalmente en el país, y acreditar su existencia y representación legal;

$\checkmark$ Manifestar bajo gravedad de juramento que el importador, sus representantes o socios, han sido sancionados con cancelación de autorización para el desarrollo de su actividad, ni penalmente durante los últimos 5 años. Del mismo modo no tener ninguna otra exigencia pendiente con la DIAN

2. Se deberá señalar el uso o destinación que se dará a la mercancía importada. La licencia de importación tendrá una vigencia de $\operatorname{dos}(2)$ años.

En Colombia para constituir, declarar y probar el derecho a explorar y explotar yacimientos mineros de propiedad del estado, se debe obtener un contrato de concesión el cual se celebra entre una persona natural y/o jurídica y el Estado para efectuar, los estudios, trabajos y 
obras de exploración de minerales de propiedad estatal que puedan encontrarse dentro de una zona determinada. Dichos minerales se explotan en los términos y condiciones establecidos en la ley (Código de Minas). El titulo Minero Consta de tres etapas, exploración, construcción y montaje, y explotación, cada una con sus obligaciones, ambientales jurídicas y económicas.
La legislación vigente establece una amplia regulación en lo que obedece a las licencias ambientales en el país, importante tenerlo en cuenta ya que por medio de ellas se trata de regular las posibles afectaciones que pueda tener el medio ambiente a raíz de diferentes actividades económicas, para nuestro caso específico la minería ilegal y la disposición final de la maquinaria empleada en ella.

\section{Figura 1 Licencias Ambientales}

Se representa de manera ilustrada los distintos escenarios a nivel normativo que regulan lo relacionado a licencias ambientales.

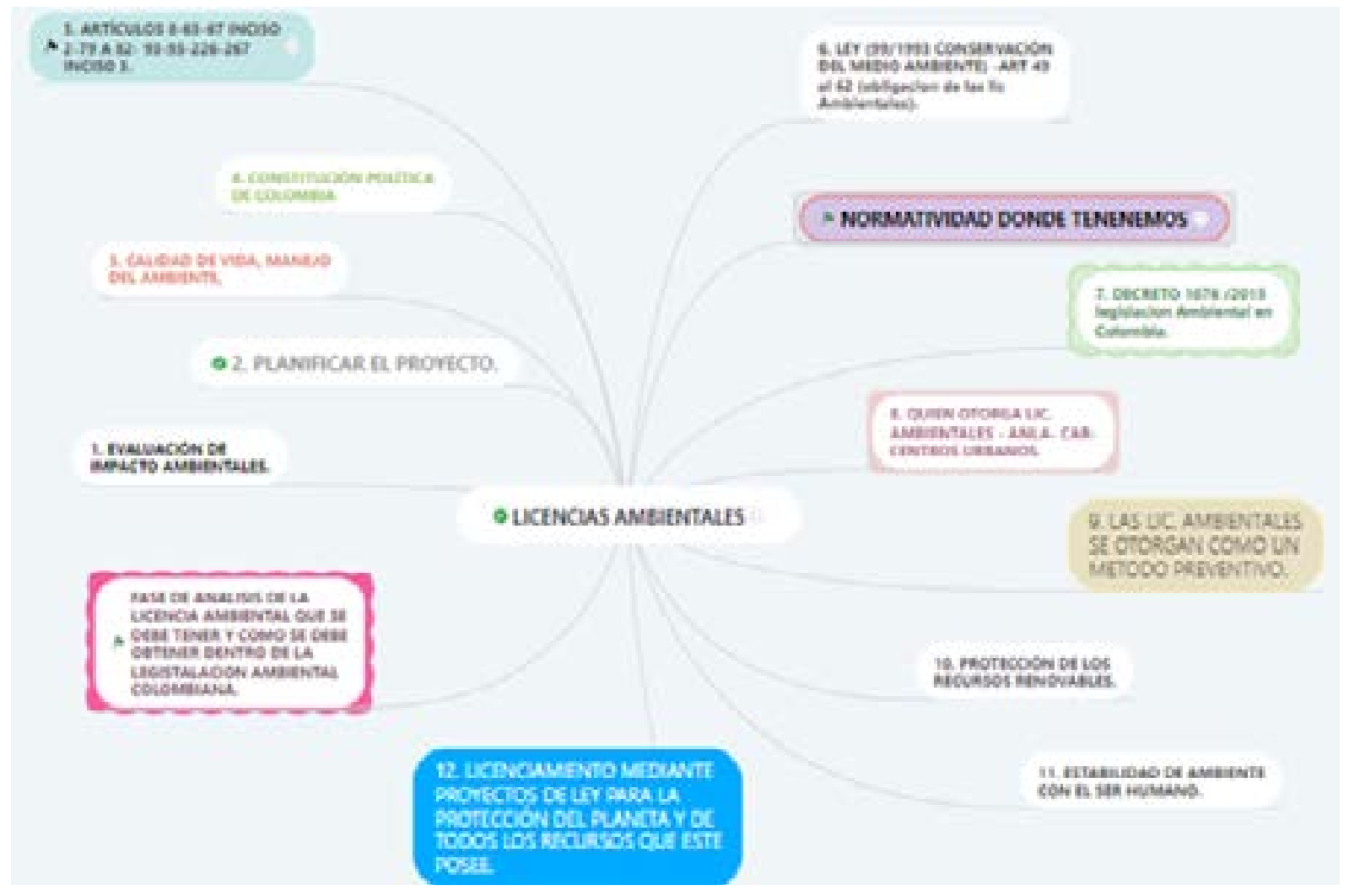

Fuente: (Lopez, 2019)

Producto de un análisis normativo acerca de las reglas y procedimientos que orientan el proceso de disposición de la maquinaria, equipos e insumos utilizados en la minería ilegal y de acuerdo al artículo 93 de la Constitución Política en el cual se consagra a los tratados y convenios internacionales como parte integral del marco legal, es necesario hacer referencia a la Decisión 774 del 3 de mayo de 2012 en la cual la Comunidad Andina de Naciones consagra la política para la lucha contra la minería ilegal.

De esta decisión y como desarrollo del objeto investigativo se resalta el articulo 5 el cual habla de medidas de prevención y control y el artículo 6 acerca de los procedimientos de decomiso 
incautación destrucción e inutilización de la maquinaria.

Mediante Decreto 2235 de 2012 se reglamenta el articulo 6 ibidem y se establece de manera particular el procedimiento para la destrucción de la maquinaria función llevada a cabo por la policía nacional.

Sin embargo, la presente investigación cuestiona que la destrucción deba ser la única opción para combatir la minería ilegal y es por eso que las medidas de seguimiento y control planteadas a continuación tienen por objeto darle a la maquinaria incautada un nuevo rumbo para lo cual se puede contar con el apoyo de la Dirección de Impuestos y Aduanas Nacionales (DIAN).

1. No se cuestiona los procedimientos y actividades mediante los cuales la policía nacional logra intervenir las explotaciones mineras ilegales $y$ las consecuentes incautaciones de maquinaria.

2. En esta etapa del proceso en el que normalmente se daría aplicación a lo preceptuado en el Decreto 2235 de 2012, pero el desarrollo o metodología planteada en la presente investigación no contempla la destrucción como una medida totalmente efectiva sin antes haber contemplado otras opciones más benéficas para la comunidad y el medio ambiente.

3. Hecha la incautación normalmente esta se incinera o se explota lo cual produce serias afectaciones al medio ambiente no solo en el lugar si no también en poblaciones cercanas al lugar de los hechos. Ya que se genera contaminación atmosférica afectaciones de fauna y flora perdida de la capa vegetal y del suelo y contaminación del recurso hídrico por el cual se perjudican las comunidades que se encuentra cerca al lugar de la destrucción de la maquinaria ilegal.
4. Deben contemplarse otras opciones como las que consagra el artículo 5 de la Decisión 774 de 2012. Concretamente se plantea dejar en disposición de la DIAN esta maquinaria de acuerdo a lo reglado en el Decreto 390 de 2016 por medio del cual se establece la regulación aduanera en cabeza de la DIAN.

5. El artículo 637 del mencionado Decreto establece lo siguiente "Formas de disposición. La Dirección de Impuestos y Aduanas Nacionales podrá disponer de las mercancías decomisadas o abandonadas a favor de la Nación, mediante la venta, donación, asignación, destrucción y/o gestión de residuos, chatarrización y dación en pago".

“Los medios de transporte aéreo, marítimo o fluvial y la maquinaria especializada podrán entregarse en comodato o arrendamiento a las entidades de derecho público, aunque su situación jurídica no se encuentre definida".

6. El artículo 8 de la Resolución 000042 del 2016 Reglamento el Decreto 390 del 2016 en lo concerniente al procedimiento de donación, teniéndose como requisitos los siguientes:

Aceptación escrita y expresa por parte del representante legal de la entidad interesada en adquirir los bienes.

$\checkmark$ Anexar los documentos que acrediten la capacidad para comprometer y obligar a la entidad beneficiaria de la donación.

7. Se tendrá preferencia a las entidades mencionadas en el artículo 53 de la Ley 1762 de 2015 que es su parágrafo 2 establece de manera muy clara que en los términos del Estatuto Aduanero, la Dirección de Impuestos y Aduanas Nacionales tiene la posibilidad de donar la mercancía aprehendida, decomisada o abandonada a favor de la nación a través de entidades del orden nacional, departamental o municipal, fuerza pública y organizaciones 
no gubernamentales sin ánimo de lucro, encargadas de programas de salud y bienestar social, educación, prevención y atención de desastres, entre otras actividades.

Se observa que la legislación vigente ya establece un procedimiento por medio del cual la maquinaria minera incautada no necesariamente debe destruirse ni generar las consecuencias mencionadas anteriormente, si no que estas pueden emplearse en favor de distintas entidades de la nación que lo que buscan es el bienestar social.

Si bien es cierto estas disposiciones no se han contemplado actualmente debido al reto que presenta debido a los lugares en donde se decomisa y su gran tamaño, no por lo mismo quiero decir esto sea imposible.

Se evidencia esta situación claramente cuando en la aplicación de instrumentos consistentes en entrevistas realizadas a funcionarios expertos de la Agencia Nacional de Minería Colombiana y la Policía Nacional, se pueden distinguir dos situaciones. Por un lado, se afirma por parte de los ingenieros de minas Egberto David Torres Jiménez (Torres, 2020) y Alcides Perales Campillo (Perales, 2020) afirman o dejan entrever en primera medida que la alternativa de no destruir la maquinaria y disponerla en donación, puede ser una muy buena opción para darle un tratamiento distinto a los procesos de intervención y erradicación de la minería ilegal, reconociendo de igual forma que el impacto ambiental se reduciría, igualmente reconocen las dificultades que pueden suponer por parte de los servidores públicos ya sean uniformados de la Policía Nacional y de la fuerza pública en general, además de aquellos funcionarios adscritos a entidades como la Agencia Nacional de Minería o la Dirección de Impuestos y Aduanas Nacionales, puedan acceder a los sitios donde se encuentra la maquinaria y disponer de ella tal como la alternativa lo plantea, esto debido a las condiciones geográficas y de orden público, afirmación respaldada por los uniformados Teniente Coronel Jhon Robert Chavarro Romero (Chavarro, 2020) y el Intendente Luis Carlos Vanegas (Vanegas, 2020) Se recalca también por parte de los funcionarios que incluso la destrucción de la maquinaria no supone el fin de la explotación ilegal en determinada zona ya que esta puede ser recuperada por parte de los actores ilegales quienes aprovechan que la fuerza pública se retira de la zona intervenida y restablece el funcionamiento dependiendo de ciertas condiciones de la maquinaria, generando de tal modo que la ilegalidad persista.

\section{DISCUSIÓN DE RESULTADOS}

La labor investigativa llevada a cabo en el presente artículo arroja varios planteamientos a tener en cuenta con el propósito de llevar a cabo las medidas de control y seguimiento con respecto a la maquinaria utilizada en los procesos de extracción minera ilegal. Se puede observar partiendo del análisis normativo que la Policía Nacional de Colombia desde hace ya varios años se encuentra comprometida en la lucha contra este ilícito el cual a pesar de los esfuerzos y datos aportados a distintas entidades del orden nacional no permiten evidenciar una disminución notable en la comisión de este tipo de conductas, de igual forma se tiene que el procedimiento estándar a la hora de realizar una intervención es el de la destrucción de la maquinaria al ser la opción más viable por las razones mencionadas anteriormente. Si bien es cierto el marco normativo que existe actualmente en Colombia apoyado desde la Decisión 774 de la Comunidad Andina de Naciones permite contemplar otras alternativas a la destrucción, estas no se aplican debido a las dificultades que presentan, para el caso en concreto la donación de esta maquinaria supondría el involucramiento de una entidad como lo es la Dirección de Impuestos y Aduanas Nacionales la cual si bien es cierto tendría competencia cuando se trata de la importación al 
país de este tipo de maquinaria, encontraría muy limitado su campo de acción cuando la misma ya se encuentra en su lugar de destinación es decir en los puntos donde se realizan las extracciones mineras ilegales, y donde la Policía Nacional lleva a cabo sus procedimientos.

Las dificultades planteadas suponen un reto mayúsculo para la aplicación de la alternativa de donación, generan que se deban contemplar otras opciones para el tratamiento del impacto ambiental y pasivos ambientales ocasionados con la destrucción de la maquinaria analizando la posibilidad de la aplicación de la normatividad vigente en el manejo integral de los residuos peligrosos consagrados en el Decreto 1076 del 2015 a lo cual garantice la disminución de los agentes contaminantes tales como aceites, hidrocarburos, combustibles, residuos sólidos, que dan origen al deterioro ambiental de los factores bióticos y abióticos en donde se realizan la intervención por parte de la Policía Nacional, de igual manera poniendo en riesgo la salud pública de las poblaciones que se encuentran aledañas al área de influencia. Se aclara que no se descarta del todo la opción o la viabilidad de poner disponer de esta maquinaria para donación ya que la explotación minera en el país se presenta o se manifiesta desde varios ámbitos, condiciones, ubicaciones que generan que la explotación ilegal que se presenta en parajes tan lejanos como la selva chocoana, a la que se presenta en los departamentos costeros del país. Se quiere dejar en claro con esto que si bien es cierto hay zonas en las que es mejor destruir la maquinaria evitando riesgos innecesarios, también lo es que en otras poblaciones esta labor pueda ser mucho más sencilla de llevar a cabo.

Se manifiesta por parte de los funcionarios encargados de llevar a cabo estos procedimientos de intervención que la afectación no es solo de carácter ambiental, aunque si se trata del principal aspecto tratado en el presente artículo, no se puede dejar de lado que el flagelo también es de carácter social, presentándose situaciones tales como la prostitución el desplazamiento forzado, la trata de personas y factores económicos que en general dificultan la aplicación de las medidas contempladas para mitigar los impactos ambientales.

\section{CONCLUSIÓN}

Actualmente en Colombia se han venido manejando distintas políticas y programas para tratar de mitigar e incluso erradicar la minería ilegal ya que este ha sido de los factores que han puesto un mayor reto para el estado a la hora de garantizar las condiciones mínimas necesarias para que las personas en su totalidad puedan gozar de libertades y derechos tal cual se consagran en la Constitución Política de 1991. especialmente el de un medio ambiente sano. Yes que la minería ilegal mueve una gran cantidad de dinero el cual es aprovechado por organizaciones ilegales para seguir financiando sus actividades ilícitas en las cuales se ven involucradas un gran número de personas a lo largo y ancho del país de manera directa e indirecta, sin lugar a dudas el hecho de que este sea un negocio tan rentable genera que se persista en su consumación y por lo tanto en los aspectos negativos que en él se derivan especialmente el impacto ambiental. Como es de conocimiento gran parte de estas explotaciones se ubican las fuentes hídricas las cuales suministran el vital líquido a distintas poblaciones del país, a pesar de que la Policía Nacional con la destrucción de la maquinaria busca frenar esta afectación, indirectamente a la contaminación. Puede considerarse es un mal necesario el cual tiene como propósito acabar con un impacto mayor, pero de igual forma se ha planteado la necesidad de tratar de darle un planteamiento distinto a esta problemática.

Tal como se planteó a lo largo del articulo y a pesar de las dificultades la alternativa de donar 
la maquinaria resulta ser una opción bastante innovadora ya que no solo generaría reducir casi en su totalidad el daño que ocasiona al medio ambiente la destrucción de la maquinaria sino que además esta puede ser destinada a distintas entidades del orden nacional, departamental o municipal que puedan emplearla en otro tipo de labores o trabajos a favor de las comunidades ya que esta seria correctamente ingresada al patrimonio del estado a través de los protocolos y procedimientos de incautación que contemplan la Dirección de Impuestos de Aduanas Nacionales en el ejercicio de sus funciones. Todo lo anterior de la mano a que las condiciones tanto geográficas como de orden público lo permitan.

De igual forma y entendiendo los riesgos y dificultades que plantea la ejecución puesta en marcha de esta alternativa se contempla en la medida de lo posible la aplicación en el sitio de intervención de los protocolos y procedimientos señalados para el tratamiento manejo y control de las sustancias que emplean ese tipo de maquinarías para su funcionamiento. Si bien es cierto no podrían disponerse de ellas en su totalidad si se puede mermar el riesgo y afectación que generan estos derivados químicos que se esparcen en la geografía cuando se explota una máquina.

En el transcurso del proceso investigativo pudo observarse y como claramente se planteó desde un principio, que la labor de protección y cuidado del medio ambiente no es solo deber de la Policía Nacional cuando interviene las explotaciones ilegales, si no que en ella confluyen otras instituciones que de una u otra forma deben cumplir con el mismo objetivo y es por medio de la articulación interinstitucional que las alternativas planteadas en el presente artículo tienen validez y pueden ejecutarse adecuadamente. No se deja de lado la importante labor llevada a cabo y el riesgo que asumen los uniformados de la policía nacional en la prestación del servicio y de manera particular, dejando en claro que ante la inquietud elevada por esta institución el trabajo debe ser mancomunado y el mismo se reflejara en la población que podrá observar cómo la policía se esfuerza no solo en limitarse al espacio de intervención sino que además y derivado del cumplimiento de este deber se contemplan otras alternativas que benefician en lo que respecta al cuidado del medio ambiente y la salud pública.

\section{PROPUESTA}

\section{ALTERNATIVAS PARA LA MAQUINARIA Y/O SUS DERIVADOS, INCAUTADOS EN LA MINERÍA ILEGAL}

\section{Objetivo}

\section{Objetivo General}

Identificar un plan de seguimiento, control y disposición final para la maquinaria utilizada en los procesos de extracción minera, especialmente la incautada por la Policía Nacional de Colombia en el contexto de su misión institucional de cuidado y protección del medio ambiente articulando esfuerzos con aquellas instituciones enfocadas al mismo objetivo.

\section{Fases}

\section{Fase De Preparación}

Acceder a información sobre el tema del uso de maquinaria en la minería ilegal solicitándole a entidades de orden público tal como UNIMIL, Dirección de Carabineros de la Policía Nacional.

\section{Fase De Delimitación}

Entrevistas con profesionales capacitados en los temas de investigación y el uso de maquinaria en las actividades de extracción minera. Identificar factores asociados a las actividades ilegales en la extracción minera. 


\section{Fase Analítica}

Realizar una validación y reducción de datos obtenidos. Ejecución de transformación de datos obtenidos. Obtención de resultados y verificación de los mismos.

\section{Fase Informática}

Elaboración del artículo, Informe de la investigación, Validación del artículo, Publicación.

\section{RESULTADOS}

Se logro identificar alternativas con respecto al uso o disposición final de la maquinaria utilizada en los procesos ilegales de explotación minera, incluidos sus derivados. En primer lugar, la donación como una opción contraria al procedimiento llevado a cabo actualmente por la Policía Nacional. En segundo lugar, que se contemple la opción por parte de las autoridades encargadas de hacer las intervenciones de aplicar en el mismo sitio los protocolos y procedimientos para el manejo de sustancias peligrosas o contaminantes.

\section{REFERENCIAS BIBLIOGRÁFICAS}

Agencia Nacional de Mineria. (2014). Obtenido de https://www.anm.gov.co/sites/ default/files/NormativaConceptosJurid icos/20141200387641-destruccion-demaquinaria-pesada.pdf

Chavarro. (Agosto de 2020). Teniente Coronel Jhon Robert Chavarro Romero.

Colombia, M. d. (30 de 10 de 2012). www. presidencia.gov.co. Obtenido de www.presidencia.gov.co: http:// wsp.presidencia.gov.co/Normativa/ Decretos/2012/Documents/ OCTUBRE/30/DECRETO\%202235\%20 DEL\%2030\%20DE\%200CTUBRE\%20 DE\%202012.pdf
Colombia, M. d. (30 de 10 de 2012). www. presidencia.gov.co. Obtenido de http://wsp.presidencia.gov.co/ Normativa/Decretos/2012/Documents/ OCTUBRE/30/DECRETO\%202235\%20 DEL\%2030\%20DE\%200CTUBRE\%20 DE\%202012.pdf

Colombia, P. N. (22 de 05 de 2019). www.policia. gov.co. Obtenido de www.policia.gov. co: https://www.policia.gov.co/noticia/ gran-ofensiva-contra-extraccion-ilicitaminerales-y-uso-del-mercurio

Corpoamazonia. $(01$ de 11 de 2018). www. corpoamazonia.gov.co. Obtenido de www.corpoamazonia.gov.co: http://www.corpoamazonia.gov.co/ images/2018/Consultas/20181207_ tecnico.pdf

Decreto 2235. (2012). Obtenido de https://www. anm.gov.co/?q=content/decreto-2235de-2012

Informe especial de la Contraloria (Mineria Ilegal). (2013). Obtenido de https://www.contraloria.gov.co/ documents/20181/198738/SeparataMineria-Ilegal.pdf/4d3d5cbe-4bda430a-831e-ef2f6bbf5d0d?version=1.0

Informe Preventivo Minería Ilegal, Procuraduría General de la Nación. (2016). Obtenido de https://www.procuraduria.gov. co/portal/media/file/MINERIA $\% 20$ ILEGAL\%20EN\%20COLOMBIA\%20\%20 DOCUMENTO.pdf

Instituto Amazónico de investigación Cientificas. (Noviembre de 2018). Obtenido de http://www.corpoamazonia.gov.co/ images/2018/Consultas/20181207_ tecnico.pdf 
Investigación Cualitativa . (2007). Obtenido de http://www.scielo.org.pe/ scielo.php?script=sci_arttext\&pid =S1729-48272007000100008

La investigacion cualitativa una discusion presente . (2007). Obtenido de https://www.redalyc.org/articulo. oa? id $=68601308$

La mineria sin control de la defensoria del pueblo. (2015). Obtenido de https:// www.defensoria.gov.co/public/pdf/ InformedeMinerla2016.pdf

Las rutas del oro ilegal. (2015). Obtenido de https://cpalsocial.org/documentos/209. pdf

Lopez, J. (2019). Javier Lopez Licencias Ambientales. Obtenido de https:// www.mindmeister.com/es/945202325/ licencias-ambientales)

Naciones, C. A. 103 de 05 de 2012). www. cancilleria.gov.co. Obtenido de www. cancilleria.gov.co: https://www. cancilleria.gov.co/sites/default/files/ decision_744.pdf

Perales. (Agosto de 2020). Ingenierio de Minas.

Policia. (22 de 22 de 2020). www.policia.gov. co. Obtenido de https://www.policia. gov.co/noticia/gran-ofensiva-contraextraccion-ilicita-minerales-y-uso-delmercurio

Sentencia T 445 de Agosto. (2016). http:// www.humboldt.org.co/images/ documentos/2-diagnstico-actividadminera-y-explotacin-ilicita-expertos. pdf.

Sistema unico de informacion normativo. (2012). Obtenido de http://www.suin-juriscol. gov.co/viewDocument.asp?id=1808886
Torres. (Agosto de 2020). Ingenierio de Minas.

Torres Jimenez, P. L. (14 de Abril de 2015). Repositorio digital universidad nacional de lojal. Obtenido de https://dspace.unl. edu.ec/jspui/handle/123456789/10492

Vanegas. (Agosto de 2020). Intendente Luis Carlos Vanegas. 